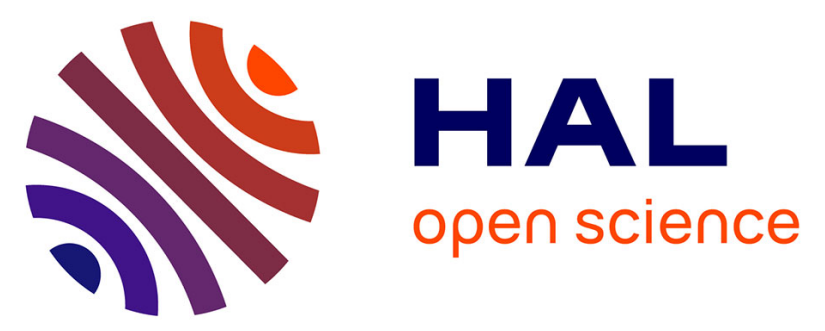

\title{
Vinyltriethoxysilane-functionalized starch nanocrystals as Pickering stabilizer in emulsion polymerization of acrylic monomers. Application in nanocomposites and pressure-sensitive adhesives
}

\author{
Emna Ben Ayed, Albert Magnin, Jean-Luc Putaux, Sami Boufi
}

\section{To cite this version:}

Emna Ben Ayed, Albert Magnin, Jean-Luc Putaux, Sami Boufi. Vinyltriethoxysilane-functionalized starch nanocrystals as Pickering stabilizer in emulsion polymerization of acrylic monomers. Application in nanocomposites and pressure-sensitive adhesives. Journal of Colloid and Interface Science, 2020, 578, pp.533-546. 10.1016/j.jcis.2020.05.011 . hal-02897088

\author{
HAL Id: hal-02897088 \\ https://hal.science/hal-02897088
}

Submitted on 29 May 2021

HAL is a multi-disciplinary open access archive for the deposit and dissemination of scientific research documents, whether they are published or not. The documents may come from teaching and research institutions in France or abroad, or from public or private research centers.
L'archive ouverte pluridisciplinaire HAL, est destinée au dépôt et à la diffusion de documents scientifiques de niveau recherche, publiés ou non, émanant des établissements d'enseignement et de recherche français ou étrangers, des laboratoires publics ou privés. 


\section{Vinyltriethoxysilane-functionalized starch nanocrystals as Pickering stabilizer in emulsion polymerization of acrylic monomers. Application in nanocomposites and pressure-sensitive adhesives.}

Emna Ben Ayed ${ }^{\mathrm{a}}$, Albert Magnin $^{\mathrm{b}}$, Jean-Luc Putaux ${ }^{\mathrm{c}}$, Sami Boufi $^{\mathrm{a}^{*}}$

${ }^{a}$ University of Sfax - LMSE - Faculty of Science - BP 802 - 3018 Sfax, Tunisia

bUniv. Grenoble Alpes, CNRS, Grenoble INP, LRP, F-38000 Grenoble, France 'Univ. Grenoble Alpes, CNRS, CERMAV, F-38000 Grenoble, France

\footnotetext{
* Corresponding author: sami.boufi@fss.rnu.tn
}

Published in: Journal of Colloids and Interface Science 578 (2020), 533-546 DOI: $10.1016 /$ j.jcis.2020.05.011 


\begin{abstract}
Emulsion polymerization provides a sustainable way to produce latex polymers for coatings and adhesives thanks to the use of water as a dispersion medium. This synthesis approach can be even more attractive if synthetic surfactant can be replaced by biobased solid particles as a stabilizer, through what is known as a "Pickering effect". Herein, latex dispersions with solid content up to $35 \mathrm{wt} \%$ were successfully produced by emulsion polymerization using starch nanocrystals (SNCs) as a sole stabilizer and $\mathrm{H}_{2} \mathrm{O}_{2} /$ citric acid as a redox-initiator. The effect of the SNC modification with vinyltriethoxysilane (VTES) on the colloidal properties of the polymer dispersion and performance of the resulting nanocomposite film were investigated. As an application of this approach, pressure-sensitive adhesive (PSA) dispersions have been prepared via Pickering emulsion polymerization in the presence of $8 \mathrm{wt} \%$ SNCs. The use of VTES-SNCs has a beneficial impact on the performance of PSAs with improved peel strength and wettability. The possibility to use SNCs as a stabilizer to replace synthetic surfactants in emulsion polymerization opens new avenues for the application of SNCs as biobased Pickering stabilizers to produce latex for coatings, adhesives, inks, and textiles.
\end{abstract}

Keywords: emulsion polymerization, starch nanocrystals, Pickering emulsion, stability. 


\section{Introduction}

Biobased nanomaterials deriving from renewable resources have emerged as a new class of nanoparticles (NPs) with increasing interest at both academic and industrial levels. Cellulose, lignin, starch, and chitin constitute different resources for the production of biobased NPs with different morphologies, including long flexible or rodlike fibrils, and platelet-like particles. Methods of production, physical and chemical properties of biobased nanoparticles and their potential use in advanced functional nanomaterials have been outlined in several reviews [1-2]. Beside their sustainability, the absence of safety concerns pointed out so far, at least for nanocellulose [3], has facilitated their handling and production. Nanocellulose, chitin nanofibrils, and starch nanoparticles have been reported to be inoffensive to humans, non-irritating to skins, harmless to aquatic organisms and fully biodegradable once disposed of [4].

Starch is an interesting and cheap source for the production of NPs with a size within the 20-300 $\mathrm{nm}$ range and exhibiting a lamellar or spherical morphology. Although more recent than nanocellulose and nanochitin, starch nanoparticles have attracted great interest during the last decade for their potential use as food bioactive ingredients and drug delivery carriers [5]. Starch granules are available in a rather pure form, without the need for harsh chemical purification procedures like for naturally occurring cellulose and chitin.

SNCs can be prepared from starch granules from various botanical sources [6].They are typically prepared by an acid hydrolysis that etches away the amorphous regions in native granules, resulting in a fraction with a crystallinity index significantly higher than that of the parent granules. The so-called "lintnerization" protocol consists in a mild hydrochloric acid $(2.2 \mathrm{~N} \mathrm{HCl})$ carried out at $35-36{ }^{\circ} \mathrm{C}$ during $2-6$ weeks $[7,8]$. The crystalline residue was used as a reinforcing filler in thermoplastic matrices [9]. The morphology and structure of the SNCs prepared by lintnerization of amylopectin-rich (waxy) maize starch granules were described as more or less individualized parallelepipedal 5-7 nm-thick platelets corresponding to the crystalline lamellae formed by the association of amylopectin side branches into parallel arrays of double helices [10]. The main limitation of the lintnerization procedure is the extended time and the rather low yield (about 5\% with waxy maize starch granules). An alternative method to prepare so-called "Nägeli amylodextrins" consists in using sulfuric acid instead of $\mathrm{HCl}$. Angellier et al. used a response surface methodology to optimize the protocol. The authors obtained a yield of $15 \mathrm{wt} \%$ by hydrolyzing waxy maize starch granules during 5 days at $40{ }^{\circ} \mathrm{C}[11]$. They also showed that the platelet morphology of the resulting SNCs was 
similar to that obtained by mild $\mathrm{HCl}$ hydrolysis [12]. These SNCs were used as reinforcing filler in acrylic polymer [12], natural rubber [13] and thermoplastic starch [14] matrices.

However, given their low aspect ratio, SNCs fail to impart a substantial reinforcing effect in comparison to nanocelluloses [13]. In packaging films, SNCs have attracted interests as additives to improve barrier properties. The platelet-like shape of SNCs along with their crystallinity have shown to be beneficial to strongly reduce oxygen diffusion and permeability of nanocomposites [13]. Regarding nanocellulose, the most reliable processing method ensuring an effective dispersion of nanocellulose within the polymer matrix is the casting of a mixture of polymer dispersion with an aqueous suspension of nanocellulose [13]. Healthcare is another field of promising application of SNCs as a carrier for drug delivery [15]. For instance, a drug may be dissolved, entrapped, adsorbed, attached or encapsulated into the SNC aggregates, for target or protected delivery of bioactive agents [16].

Pickering emulsions are solely stabilized by solid particles adsorbed at the oil-water interface. Unlike surfactant-stabilized emulsions where the stabilization is driven by the reduction in interfacial tension, in Pickering emulsion, the stabilization is due to the reduction of the bare oil-water interface through the adsorption of solid particles, creating a physical barrier against the coalescence of the dispersed phase and blocking the Ostwald ripening process [17]. A wide range of solid particles, such as cellulose nanocrystals [20], chitin nanocrystals [21] silica beads [22], clay [23], and carbon black [24] have been reported as Pickering emulsifiers. An overview of Pickering emulsion with a focus on the field of applications and the particles/nanoparticles likely to be used have been reported in a numbers of reviews $[19,25]$.

Recently, the use of starch based Pickering emulsions have received much interest due to the broad capacity of the emulsification of SNCs to produce the oil-water emulsion [18]. A recent work has shown that the esterification of SNCs with octenyl succinic acid at a low DS degree (as low as 0.012) improves the emulsifying capacity of starch nanocrystals for stabilization of oil-in-water emulsions [19].This approach could find applications in the encapsulation of bioactive components for functional food, food product formulations and Pickering emulsion polymerization [20]. By substituting synthetic hazardous surfactants or amphiphilic polymers by SNCs, more environment-friendly and biocompatible emulsions can be produced with potential applications in food, fine chemical synthesis, cosmetics, waterborne adhesives and coatings.

In 2014, the first work on the one-step surfactant-free Pickering emulsion polymerization using a water-soluble radical initiator and SNCs as a stabilizing agent in the presence of poly(ethylene glycol) methacrylate (PEGM) as a co-monomer has been reported by our 
research group [21,22]. In this work no surface modification was carried out and PEGM was used to promote the SNC-polymer particle adhesion. This approach was shown to be doubly beneficial. On the one hand, no-surfactant was used, and on the other hand, nanocomposite films were obtained with much better mechanical properties and optical transparency than those obtained by blending the polymer emulsion with SNCs. Since then, the use of SNCs in the emulsion polymerization has been reported in several papers. For instance, expandable spherical polystyrene beads $(2-3 \mathrm{~mm}$ in diameter) were prepared through surfactant-free Pickering emulsion polymerization in water-in-oil-in-water (w/o/w) system using cross-linked starch nanoparticles as an emulsifier [22].Stable and low-viscosity latexes were successfully produced at up to $17 \mathrm{wt} \%$ SNCs loading and $42 \mathrm{wt} \%$ solids with a semi-batch emulsion polymerization protocol $[23,24]$. The main motivation of the work was to partially replace petroleum-based monomers with biobased renewable materials. However, so far, the role of SNCs in the stabilization process has not been clarified.

Trialkoxyorganosilanes are industrial additives widely used for surface modification to promote adhesion in composites and adhesives and to turn surface either to hydrophobic or hydrophilic. They have a typical structure of $\mathrm{X}-\mathrm{R}-\mathrm{Si}(\mathrm{OR}) 3$ where $\mathrm{X}$ is a non-hydrolysable organic moiety that can be reactive(e.g., methacrylic, vinylic, epoxy, amino, sulfur) or nonreactive (e.g., alkyl), - OR' is an alkoxy hydrolysable group (e.g., methoxy, ethoxy) and R is a spacer that could be either an aryl or alkyl chain. The availability of a wide choice of commercial organosilane derivatives with dual functionalities and with the possibility to react on inorganic surfaces as well as organic materials has contributed to expand the use of this class of compounds to broad technology areas including composites, coating, building, automotive and electronics, among others [25].

In the present work, we pursue our investigation concerning the use of SNCs as Pickering stabilizer in emulsion polymerization without adding any surfactant or costabilizer. With the objective to enhance the stabilization efficiency of SNCs and increase the solid content during the emulsion polymerization to meet the application requirement, vinyltriethoxysilane (VTES) was grafted onto SNCs. The effect of the silane modification on the particle size, colloidal stability and mechanical performance has been studied. A nanocomposite dispersion based on a low glass transition copolymer emulsion stabilized with SNCs was synthesized by emulsion copolymerization of ethylhexylacrylate and butylmethacrylate monomers and tested as a pressure-sensitive adhesive. Given the commercial availability of VTES and in general functional trialkoxysilane reagents, their safety to use, and the wide possibility to modify the wettability, the VTES-modified SNCs should open new perspectives to use SNCs as a 
biobased stabilizer for latex production, in replacement of synthetic surfactants. Compared to our previous report regarding the use of SNCs as a stabilizer in emulsion polymerization, the present work took advantage of a surface modification with commercial VTES to promote the adhesion to the polymer particle and enhance the stabilization efficiency of SNCs. Another motivation for this functionalization was to increase the solid content of the polymer dispersion to over $30 \mathrm{wt} \%$ so that a commercial application of the latex might be considered.

\section{Experimental}

\subsection{Materials}

Butylmethacrylate (BMA, Aldrich, $99 \mathrm{wt} \%$ ), ethylhexylacrylate, $\mathrm{H}_{2} \mathrm{O}_{2}$, citric acid, vinyltriethoxysilane (VTES) and sulfuric acid were supplied by Aldrich and used without further purification. Distilled water was used for all the polymerization and treatment processes.

\subsection{Starch nanocrystal preparation}

Starch nanocrystals were prepared by sulfuric acid hydrolysis of waxy maize starch as described in details in previous works $[26,27]$. Briefly, $5 \mathrm{~g}$ of starch was added to $3.16 \mathrm{M}$ aqueous sulfuric acid solution and left under magnetic stirring for 5 days at $40^{\circ} \mathrm{C}$ in a thermostated bath. Then the suspension was centrifuged at $10,000 \mathrm{rpm}$ to recover the SNCs. The SNCs were centrifuged repeatedly with distilled water until neutrality. A homogeneous dispersion of starch nanocrystals was obtained by using aT25UltraTurrax homogenizer for 3 min at $13,500 \mathrm{rpm}$.

\subsection{Modification of SNCs with VTES}

Freeze-dried starch nanocrystals (1 g) were dispersed in $20 \mathrm{~mL}$ toluene under sonication for $1 \mathrm{~min}$. Then, the suspension was kept under magnetic stirring for $15 \mathrm{~min}$ and $0.3 \mathrm{~g}$ of VTES was added. After stirring during $24 \mathrm{~h}$ at $80{ }^{\circ} \mathrm{C}$, the suspension was centrifuged to recover SNCs, which were washed three times with ethanol to remove adsorbed VTES. The modified SNCs were dried at $50{ }^{\circ} \mathrm{C}$ for $1 \mathrm{~h}$ under vacuum and then stored under nitrogen to avoid any risk of reaction between the vinyl groups and oxygen. Referring to the literature data and silane chemistry [25], the grafting reaction is depicted in Scheme 1. The modified SNCs will be referred to as VTES-SNCs in the following. Since SNCs remained in the form of suspension during the whole functionalization/recovering treatment, almost all the SNCs were easily recovered without significant loss of the material. 


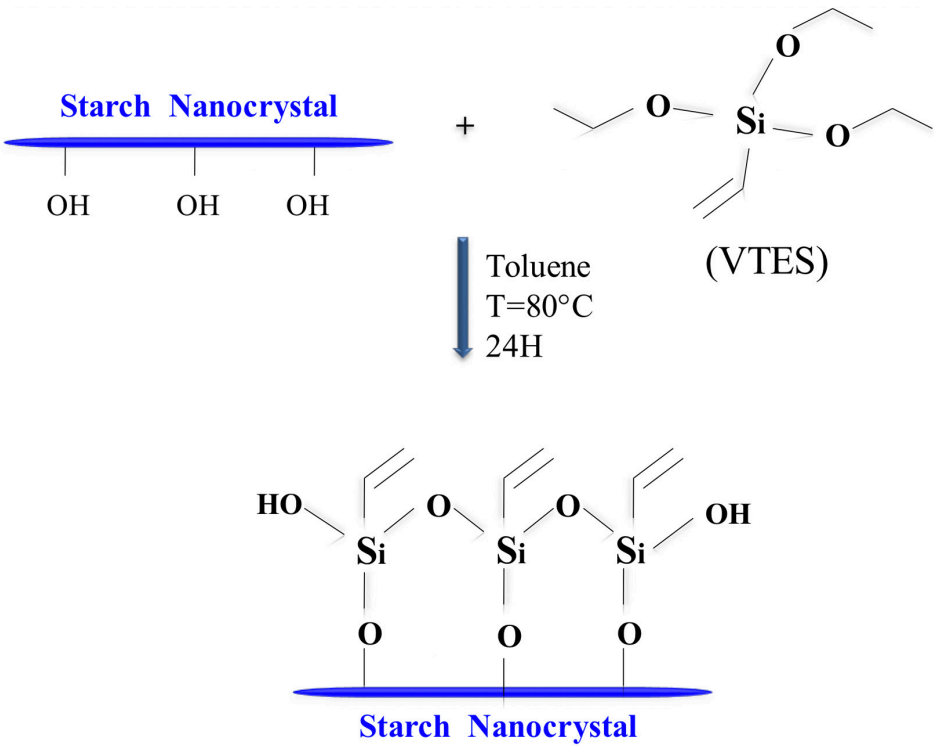

Scheme 1. Schematic illustration of the grafting of VTES onto SNCs.

\subsection{Emulsion polymerization}

The typical formulation for the emulsion polymerization of BMA in the presence of SNCs is given in Table 1. The procedure adopted is the following: SNCs were first dispersed in an appropriate volume of water so that the whole amount of water was $16 \mathrm{~mL}$, and were sonicated for $1 \mathrm{~min}$ at a 70\% amplitude (Sonics Vibracel Model CV33) to ensure an effective dispersion. Then, BMA and citric acid were added and emulsified by magnetic stirring for 5 min and followed by a 1 min sonication. After flushing with $\mathrm{N}_{2}$, the kit was placed in a heated bath at $50{ }^{\circ} \mathrm{C}$ and the polymerization was triggered by the addition of $\mathrm{H}_{2} \mathrm{O}_{2}$ during $1.5 \mathrm{~h}$. The polymerization was pursued for $2 \mathrm{~h}$ to ensure a complete consumption of the monomer. Once the polymerization was achieved, $0.1 \mathrm{~g}$ of $0.1 \% \mathrm{NaN}_{3}$ was added as a preservative to prevent bacterial growth. In another set of experiments, the SNC suspension was first heated at $70{ }^{\circ} \mathrm{C}$ for $3 \mathrm{~h}$ to completely disrupt the crystalline structure and the emulsion polymerization was carried out following the procedure previously described. In the different polymerization runs, the amount of SNCs will be expressed in terms of a percentage with respect to the monomer content.

For all polymerization runs, the end of the polymerization reaction was checked by the determination of the conversion degree using the gravimetric method. In all cases, a conversion degree exceeding $98 \%$ was reached within a $3 \mathrm{~h}$ polymerization. 
Table 1. Emulsion polymerization of BMA in the presence of SNCs as sole stabilizer $\left(50{ }^{\circ} \mathrm{C}\right)$.

\begin{tabular}{cc}
\hline Constituent & Composition $(\mathrm{g})$ \\
\hline Water & 17 \\
BMA & $4-7$ \\
$\mathrm{H}_{2} \mathrm{O}_{2}$ & 0.15 \\
$\mathrm{AA}$ & 0.15 \\
$\mathrm{SNC}$ & 0 to 0.3 \\
\hline
\end{tabular}

\subsection{Polymer particle size}

The diameter of the polymer particles in the emulsion was measured at $25{ }^{\circ} \mathrm{C}$ using a Malvern Nano ZS Zetasizer instrument at a fixed scattering angle of $173^{\circ}$. The dispersion was diluted to about $5 \mathrm{wt} \%$ with distilled water before starting the measurements. The particle size was expressed as a Z-average corresponding to the diameter of an equivalent hard sphere having the same diffusion coefficient as that of the particle being measured. The measurement provides also indication about polydispersity of the sample through the polydispersity index (PDI). Each measurement was performed in triplicate and the values were averaged to obtain the mean particle diameter.

\section{6. $\zeta$-Potential measurement}

The $\zeta$-potential was measured at $25{ }^{\circ} \mathrm{C}$ using a laser Doppler electrophoresis apparatus (Malvern Nano-Zetasizer ZS, UK). The sample consistency in water was set to $0.01 \%(\mathrm{w} / \mathrm{v})$. The measurements were performed three times for each sample.

\subsection{Fourier-transform infrared spectroscopy (FTIR)}

FTIR spectra were recorded on a Perkin Elmer FT-IR Spectrum 100 in absorbance mode. $\mathrm{KBr}$ pellets containing $20 \mathrm{mg}$ of material and $200 \mathrm{mg}$ of $\mathrm{KBr}$ were prepared under the same condition and their FTIR spectra recorded with $\mathrm{KBr}$ as background.

\subsection{Field-emission scanning electron microscopy (FE-SEM)}

Small amounts of freeze-dried latex powder were deposited on silicon wafers and coated with a 2-3 nm-thick carbon layer by ion sputtering. Images were recorded using an in-lens secondary electron detector in ZEISS Ultra 55 and Gemini SEM 500 microscopes equipped with field-emission (FE) guns and operated at a $2-5 \mathrm{kV}$ acceleration voltage. 


\subsection{Nanocomposite film preparation}

The nanocomposite films were prepared by casting of the PBMA / SNC dispersion in a Teflon mold and storing $40^{\circ} \mathrm{C}$ until water evaporation was completed.

\subsection{Pressure-sensitive adhesive (PSA)}

A PSA dispersion based on ethylhexylacrylate/BMA (85/15 wt\%) was prepared in the presence of $8 \mathrm{wt} \%$ SNCs or VTES-SNCs, following the procedure previously described. In the absence of SNCs, sodium dioctylsulfanosuccinate (3 $\mathrm{wt} \%$ with respect to monomer content) was used as an anionic surfactant to ensure stabilization and produce stable latex dispersion.

\subsection{Dynamic mechanical analysis (DMA)}

DMA experiments were conducted in tension mode using a PYRIS Diamond DMA (Perkin- Elmer, Waltham, MA, USA). Temperature scans were run from -20 to $100{ }^{\circ} \mathrm{C}$ at a heating rate of $2{ }^{\circ} \mathrm{C} \cdot \mathrm{min}^{-1}$, a frequency of $1 \mathrm{~Hz}$ and an amplitude of $10 \mu \mathrm{m}$. Sample were about $20 \mathrm{~mm}$-long, $5 \mathrm{~mm}$-wide and around $200 \mu \mathrm{m}$-thick.

\subsection{2. $90^{\circ}$ Peel strength and rheology analysis}

A PSA thin film with a thickness about $100 \mu \mathrm{m}$ was first prepared by coating the latex dispersion onto a PET thin film using a micrometer adjustable film applicator followed by drying in oven at $60^{\circ} \mathrm{C}$ for $2 \mathrm{~h}$. The peel strength was also measured using a universal testing machine (Instron). For peel strength tests, $25 \mathrm{~mm}$-wide strips were cut and attached to a stainless steel substrate with the help of coated rolls. The average force $(\mathrm{N})$ required to peel the strip from the substrate at an angle of $180^{\circ}$ was recorded at a peel rate of $300 \mathrm{~mm} \cdot \mathrm{min}^{-1}$ at $25^{\circ} \mathrm{C}$. Rheological properties were investigated by a controlled stress rheometer (Pro + Kinexus from Malvern) using a plate-plate configuration $(10 \mathrm{~mm}$ in diameter and a gap of $500 \mu \mathrm{m})$ at $25^{\circ} \mathrm{C}$. Firstly, an amplitude sweep in the deformation range of $0.1-100 \%$, at frequencies was carried out at a frequency of $1 \mathrm{~Hz}$ to define the linear viscoelastic region (LVE) of acrylic PSA. Then, frequency sweep from 0.01 to $10 \mathrm{~Hz}$ was performed at a strain of $1 \%$ within the linear domain where the storage modulus $\left(\mathrm{G}^{\prime}\right)$ and loss modulus $\left(\mathrm{G}^{\prime \prime}\right)$ were recorded. 


\subsection{X-ray diffraction (XRD)}

Initial waxy maize starch granules and powders of ground SNC and VTES-SNC films were left for 5 days in a chamber equilibrated at $93 \%$ relative humidity $(\mathrm{RH})$. The hydrated powders were poured into $1 \mathrm{~mm}$ (outer diameter) glass capillaries that were flame-sealed and X-rayed in a Warhus vacuum chamber using a Philips PW3830 generator operating at $30 \mathrm{kV}$ and $20 \mathrm{~mA}$ (Ni-filtered $\mathrm{CuK} \alpha$ radiation, $\lambda=0.1542 \mathrm{~nm}$ ), during $1.5-\mathrm{h}$ exposures. Twodimensional diffraction patterns were recorded on Fujifilm imaging plates, read offline with a Fujifilm BAS 1800-II bioimaging analyzer. Profiles were calculated by rotational averaging of the $2 \mathrm{D}$ patterns.

\subsection{Transmission electron microscopy (TEM)}

Droplets of ca. $0.001 \mathrm{wt} \%$ SNC or VTES-SNC suspensions were deposited onto glowdischarged carbon-coated copper grids. Prior to drying, a drop of $2 \mathrm{wt} \%$ uranyl acetate negative stain was deposited on the specimens. The stain in excess was blotted with filter paper and the remaining liquid film allowed to dry. The specimens were observed with a JEOL JEM 2100-Plus microscope operating at $200 \mathrm{kV}$. The images were recorded with a Gatan Rio 16 digital camera.

\section{Results and discussion}

\subsection{Colloidal properties of SNCs and VTES-SNCs}

SNCs were produced following the well-known sulfuric acid hydrolysis route during several days. TEM images revealed polygonal platelets with an equivalent diameter ranging between 35 and $130 \mathrm{~nm}$ for individual particles (Fig. 1A). However, a partial aggregation was observed which is likely due to drying and/or staining. DLS measurement of sonicated diluted suspension of SNCs gave a monomodal size distribution centered at around $165 \mathrm{~nm}$ and a PDI around 0.11 which is indicative of a narrow size distribution (Fig. 2A). The $\zeta$-potential of the particles was around $-30 \mathrm{mV}$ and remained negative over the whole $\mathrm{pH}$ range from 2 to 12 , which results from the presence of the sulfate groups $\left(-\mathrm{OSO}_{3}{ }^{-}\right)$at the surface of $\mathrm{SNCs}$ resulting from the esterification reaction during sulfuric acid hydrolysis. The TEM images of VTES-treated SNCs did not reveal any visible change compared to the untreated SNCs (Fig. 1C,D), which demonstrates that the treatment at $80^{\circ} \mathrm{C}$ in toluene in the absence of water did not alter the morphology or structure of the SNCs. The particle size distribution by DLS remained monomodal with an equivalent diameter around $195 \mathrm{~nm}$ and a PDI around 0.17 
(Fig. 1A). The higher particle size by DLS is likely due to the decrease in the hydrophilic character of VTES-SNCs following the grafting of VTES moiety. The $\zeta$-potential of VTESSNCs decreased to about $-16 \mathrm{mV}$, which might be due to the screening of a part of sulfate groups by the grafted VTES (Fig. 2B).
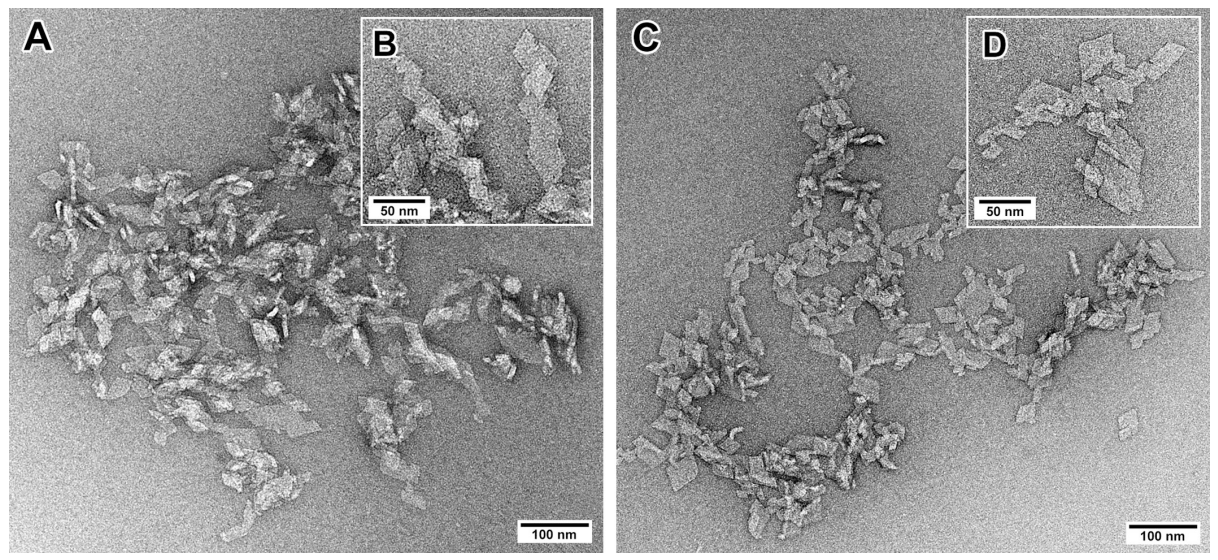

Figure 1.TEM images of negatively stained preparations of neat SNCs $(\mathrm{A}, \mathrm{B})$ and VTES-SNCs (C,D).
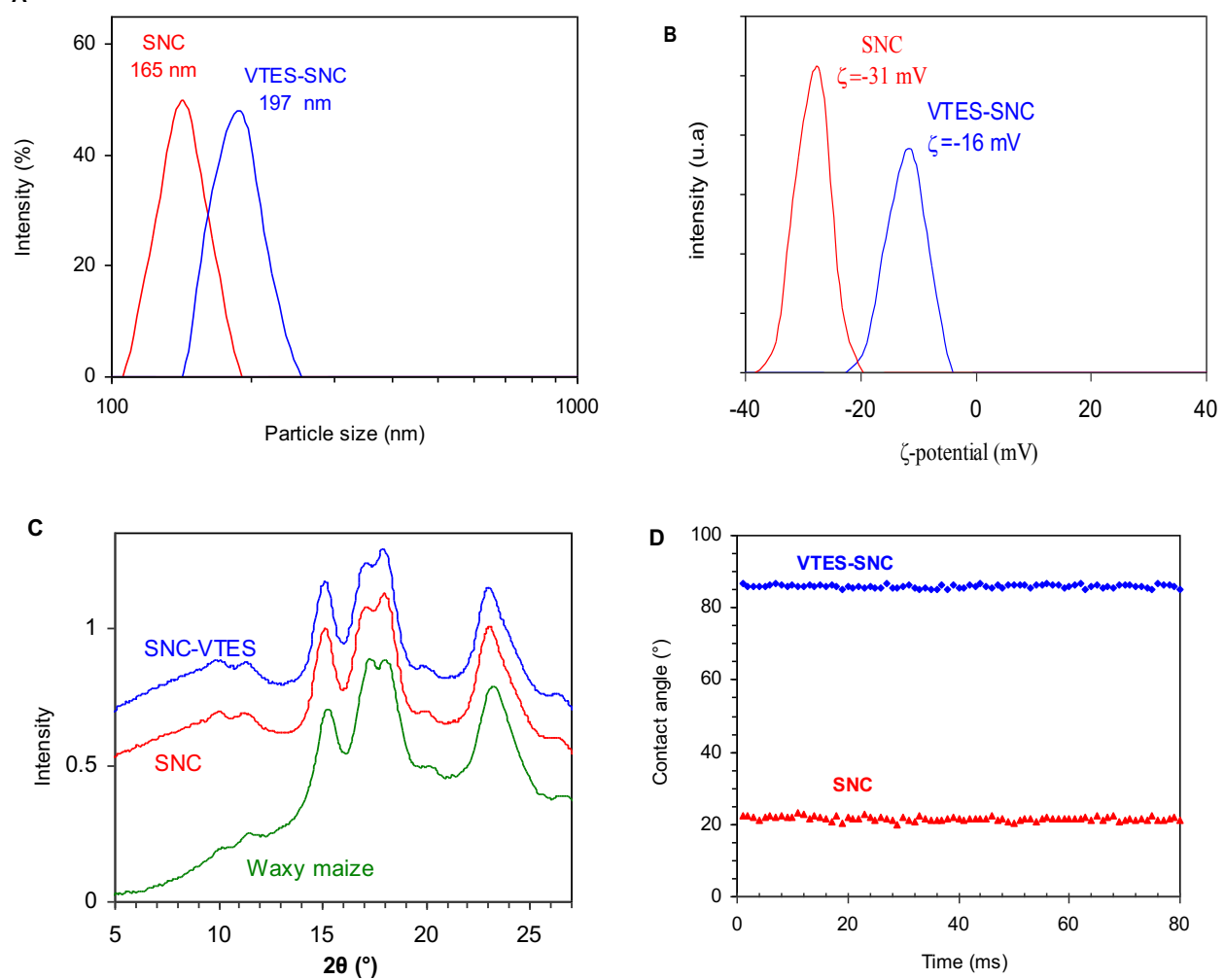

Figure 2. (A) particle diameter distribution, (B) $\zeta$-potential, (C) X-ray diffraction profiles, and (D) contact angle $v s$ time of SNCs and VTES-SNC films. 
The SNCs have an A-type crystalline structure similar to that of the parent waxy maize starch granules, with typical diffraction peaks at $2 \theta=15.5,17.2,18$ and $23^{\circ}$ (Fig. 2C). The chemical modification of SNCs did not induce any evolution either on the crystalline structure or on the crystallinity degree, suggesting that the silylation was limited to the surface of SNCs. The FTIR spectra of SNCs and VTES-SNCs recorded using the same amount of SNCs and $\mathrm{KBr}$ revealed a small evolution in the band around $1000 \mathrm{~cm}^{-1}$ (Fig. 3A). The evolution in the FTIR spectra was better outlined from the difference spectra of VTES-SNCs and that of SNC, revealing mainly a positive band around $1020 \mathrm{~cm}^{-1}$ and a negative one around $975 \mathrm{~cm}^{-1}$. The former positive band indicated that the chemical structure of VTES-SNCs generates a strong absorption band in this domain, presumably originating from $\mathrm{Si}-\mathrm{O}-\mathrm{Si}$ and $\mathrm{Si}-\mathrm{OH}$ groups grafted on SNCs [28]. The negative band observed is explained by the reduction in the surface density of the hydroxyl groups after condensation with VTES. Although the evolution in the FTIR spectra is minor, it is in agreement with the grafting of VTES according to the reaction route depicted in Fig. 3. The variation of the water contact angle from $17-22^{\circ}$ for the neat SNCs to around $85^{\circ}$ for VTES-SNCs (Fig. 2D) is another result supporting the grafting of VTES on the surface of SNCs. The low water contact angle of neat SNCs is due to the high density of hydroxyl groups on their surface along with the presence of ionic sulfate groups. The grafting of VTES on the surface masks these hydroxyl groups with a generation of apolar $\mathrm{Si}-\mathrm{CH}=\mathrm{CH}_{2}$ moieties. It should be noted that the amount of added VTES during the modification of SNCs has a strong effect on the final contact angle of VTES-SNCs. Increasing the SNC/VTES ratio over 1/0.6 made SNCs highly hydrophobic, impossible to disperse in water.

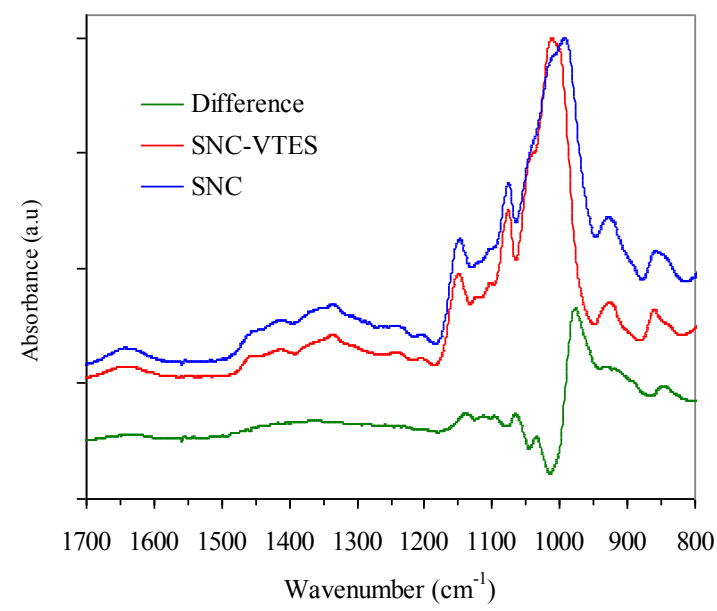

Figure 3. FTIR spectra of SNCs (blue) and VTES-SNCs (red) and the corresponding difference spectrum (green). 


\subsection{Pickering emulsion polymerization}

\subsubsection{Stability of SNCs and VTES-SNCs upon hydrothermal treatment}

Given the known sensitivity of SNCs upon heating in water, we have observed the specimens after heating dilute suspensions at $50{ }^{\circ} \mathrm{C}$ for $3 \mathrm{~h}$. The TEM images of both neat SNCs (Fig. 4A,B) and VTES-SNCs (Fig. 4C,D) show that although polygonal platelets can still be seen, compared to the initial specimens, their width appears to have decreased to some extent. It is likely that the hydrothermal treatment resulted in a partial disruption of the double helices structure of the crystalline lamellae. Angellier-Coussy at al.[29] have shown that the nanocrystals prepared by a fast sulfuric acid hydrolysis of waxy maize starch granules still contained a significant number of branching points and short branches that may act as defects in the nanocrystal. In addition, differential scanning calorimetry (DSC) thermograms of starch nanocrystals exhibit a wide endotherm suggesting that the crystallites are dissolved over a wide range of temperatures, the more "perfect" crystallites being more resistant and dissolving at a higher temperature [7]. In the present case, the TEM images show that a partial dissolution of crystallites occurred upon heating at $50{ }^{\circ} \mathrm{C}$. Fig. 4B and 4D also show that the residual platelets coexist with loose tortuous networks that resemble those formed by recrystallization of solubilized amylopectin [30]. These networks are likely formed by reassociation of the dextrins (branched or not) that are detached from the SNCs during heating, after cooling down the suspension prior to sample preparation for TEM. However, from these TEM images only, it is not possible to evaluate the relative fraction of residual crystallites and solubilized dextrins.
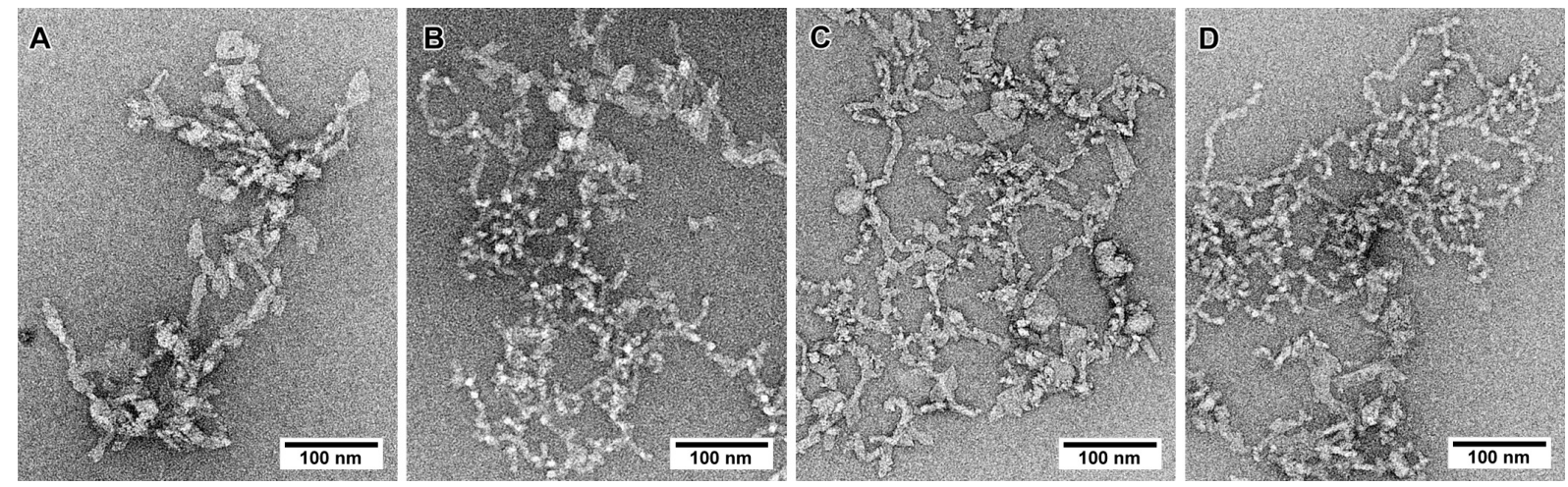

Figure 4. TEM images of negatively stained preparations from aqueous suspensions of neat SNCs $(\mathrm{A}, \mathrm{B})$ and VTES-SNCs $(\mathrm{C}, \mathrm{D})$ heated at $50^{\circ} \mathrm{C}$ for $3 \mathrm{~h}$. 

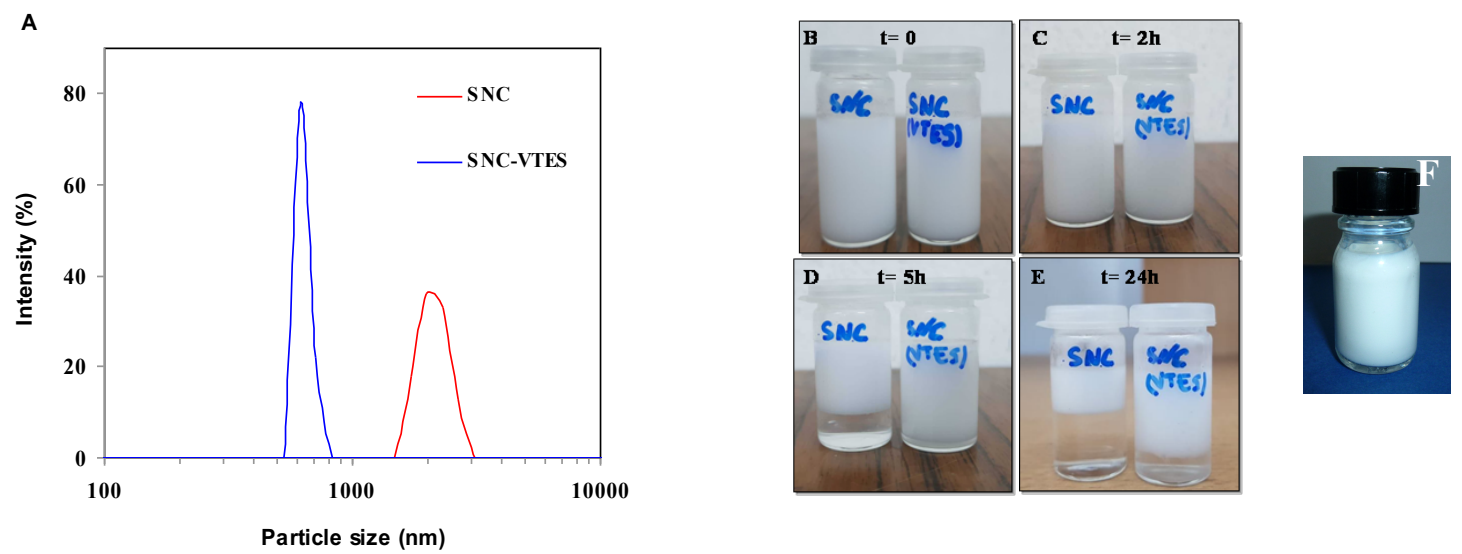

Figure 5. (A) Particle size distribution of monomer emulsion prior to the polymerization reaction;(B-E) visual aspect of the monomer emulsion after standing at different times; $(\mathrm{F})$ PBMA dispersion after emulsion polymerization.

\subsubsection{Emulsion polymerization}

The emulsion polymerization was performed in batch by mixing the monomer with SNC or VTES-SNC suspensions without any surfactant and progressive addition of initiator over $1.5 \mathrm{~h}$ at a constant temperature of $50{ }^{\circ} \mathrm{C}$. The choice of a relatively low polymerization temperature was compelled by the expected modification of SNCs upon heating in the presence of water leading to the partial dissolution of amylopectin chain segments of the lamellae. The addition of CA deemed necessary to accelerate the decomposition of $\mathrm{H}_{2} \mathrm{O}_{2}$ via a redox process [31] to provide enough hydroxyl radical to maintain satisfactory polymerization kinetics. During the first hour, the monomer remained in the form of a coarse visible droplet and the mixture rapidly became hazy after about $30 \mathrm{~min}$ of the change of turbidity (Fig. 5B-E). During the polymerization, the dispersion progressively turned milky, and the final dispersion was a milky fluid product without any significant evolution in viscosity.

Before the activation of the polymerization reaction, an emulsion of BMA was formed after the sonication treatment, the stability of which depended on the SNC content and the modification of SNCs. In the presence of neat SNCs, a phase separation was observed within 2-3h, with water decanting downward and monomer creaming upward, while the phase separation was delayed in time when modified VTES-SNCs was used as can be clearly seen in Figure 5E. Analysis of the particle size of the monomer droplet by dynamic light scattering (DLS) confirmed this trend with lower particle size of the emulsion containing VTES-SNCs (around $700 \mathrm{~nm}$ at $8 \mathrm{wt} \%$ ) in comparison of emulsion with SNCs (in the 2-3 $\mu \mathrm{m}$ range at 8 $w t \%)$. This confirmed the higher aptitude of VTES-SNCs to stabilize o/w emulsion thanks to its higher hydrophobic character than neat SNCs and their platelet morphology favoring the 
attachment of the nanoparticle on the oil phase. However, after several days, a phase separation occurred leaving the monomer layer floating on top of the water phase. This indicated that both SNCs and VTES-SNCs could not prevent the coalescence and Ostwald ripening of monomer droplets.
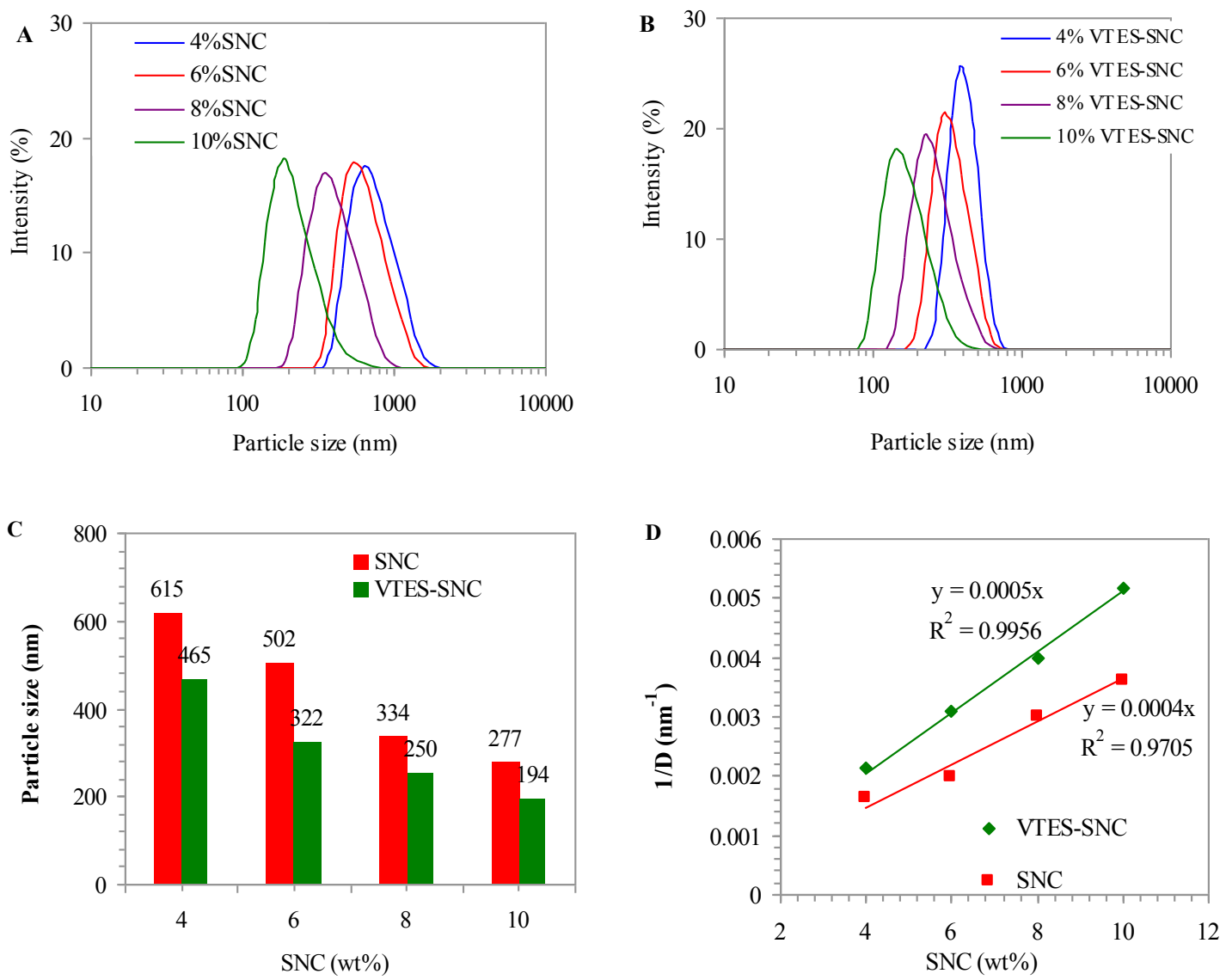

Figure 6. Particle diameter distribution for PBMA dispersions produced via Pickering emulsion polymerization in the presence of SNCs (A) and VTES-SNCs (B); (C) mean particle diameter at different SNC contents; (D) plot of inverse diameter 1/D of the polymer particles $v s \mathrm{SNC}$ content. The SNC content is expressed in $\mathrm{wt} \%$ with respect to the monomer amount.

After the polymerization reaction, all formulations with a SNC content higher than or equal to $4 \mathrm{wt} \%$ produced a stable milky latex dispersion free from any coagulum with a monomodal distribution peaking from 200 to $500 \mathrm{~nm}$, depending on the SNC content. The polymer dispersion remained stable upon storage for more than 3 months without any sign of sedimentation or phase separation. Below $4 \mathrm{wt} \%$ SNC loading, the kinetics of polymerization was slow and a marked agglomeration of polymer particles occurred resulting in the formation of coarse polymer lumps visible to the naked eye. This means that the presence of SNCs is a prerequisite to produce colloidally stable dispersions of polymer particles and that 
SNCs play a key role in the stabilization process. This could be further highlighted by looking at the evolution of the mean particle size of the dispersion with the SNC content, where a clear tendency of a decrease in particle size could be seen with increasing SNC content (Fig. 6A-C). At $4 \mathrm{wt} \% \mathrm{SNCs}$, the particle size of the latex dispersion with a monomer content of $20 \mathrm{wt} \%$ reached about $600 \mathrm{~nm}$ and decreased to about $280 \mathrm{~nm}$ when the SNC amount increased to $10 \mathrm{wt} \%$. This close dependence of the particle size on the amount of added SNCs provides a sound evidence of the aptitude of SNCs to act as a Pickering stabilizer in emulsion polymerization of acrylic monomers. This could be explained as follows: in Pickering emulsion, solid particles stabilizer are adsorbed at the surface of the dispersed phase giving rise to a physical barrier against the aggregation of suspended organic phase. The increment in the solid stabilizer content makes more particles available to be attached to the dispersed phase, which inevitably results in a reduction of their particle size. This correlation resulted in proportionality between the inverse of the diameter 1/D of the dispersed phase and the solid particle content. This relation was found to be presently valid, with a linear evolution of the inverse diameter of the polymer particles $v s$ SNC amount (Fig. 6D), confirming that the interfacial area was truly proportional to the SNC amount. This further supports the hypothesis of a Pickering stabilization during the emulsion polymerization of BMA in presence of SNCs. The larger particles in the emulsion prior to the polymerization reaction in comparison with that of the latex dispersion indicates that the monomer droplets were not the locus of the polymerization reaction and that the polymerization really occurred via a heterogeneous emulsion polymerization with a monomer acting as a reservoir to feed the polymer particles (Fig. 5A).

Interestingly, it can be seen that the latex dispersion produced with VTES-SNCs contains smaller particles than that synthesized in presence of neat SNCs. For instance, at 6 and 10 $\mathrm{wt} \%$ SNCs, the particle size reached 502 and $277 \mathrm{~nm}$, respectively, and decreased to 322 and $182 \mathrm{~nm}$ when VTES-SNCs were used. A linear tendency was also verified in the plot of 1/D vs VTES-SNC content over the whole investigated range. The lower particle size in the presence of VTES-SNCs denotes a stabilizing effect imparted by the silane groups. Two hypotheses might explain the enhancement in the stabilizing efficiency:

1) The better wettability of VTES-SNCs toward the monomer droplet/polymer particle increases the adsorption of SNCs on the dispersed phase. Actually, the particle binding energy is strongly dependent on the contact angle of water on the surface of the particles as expressed in (Eq. 1) [32]: 


$$
E=\gamma_{w p} R^{2}(1-\cos \theta)^{2}
$$

where $R, \gamma_{w p}$ and $\theta$ represent the particle radius, oil-water interfacial tension and three-phase contact angle respectively. Since the water contact angle increased from $20^{\circ}$ for neat $\mathrm{SNCs}$ to about $80^{\circ}$ for VTES modified SNCs, the energy of binding of SNCs on the monomer droplet/polymer particle notably increased, favoring a better attachment of VTES-SNCs on the dispersed phase.

2) The vinyl moiety attached to the surface of VTES-SNCs further promotes the adhesion of SNCs to the polymer particles via the copolymerization of the vinyl groups with BMA during the particle nucleation.

The key role of SNCs in the stabilization process, especially in the presence of VTESSNCs can further be emphasized by the $\zeta$-potential of the polymer dispersion at different SNC contents. In the presence of neat SNCs, the $\zeta$-potential of the polymer dispersion is close to $17 \mathrm{mV}$ over the whole range of SNC content, from 4 to $10 \mathrm{wt} \%$, meaning that the polymer particles were negatively charged (Fig. 6A). However, this value is not high enough to promote colloidal stabilization of polymer particles through electrostatic repulsion, for which the $\zeta$-potential should be higher than $25 \mathrm{mV}$ in its absolute value. The magnitude of the $\zeta$-potential of polymer particles is even lower (around -9mV) when VTES-SNCs were used (Fig. 6A). If we assume that a minimum $\zeta$-potential of $\pm 25 \mathrm{mV}$ is a prerequisite to ensure electrostatic stabilization [33], we infer that that the colloidal stability of the polymer dispersion during emulsion polymerization is truly due to a Pickering effect, rather than electrostatic repulsion. It is worth noting that in the $\mathrm{H}_{2} \mathrm{O}_{2} /$ citric acid redox system, the initiation is ensured by $\mathrm{OH}^{*}$, which did not contribute to any charge to the polymer particle. Therefore, the charges of the polymer particle results from the presence of SNCs on their surface, which explains the lower $\zeta$-potential when neat SNCs were used. 

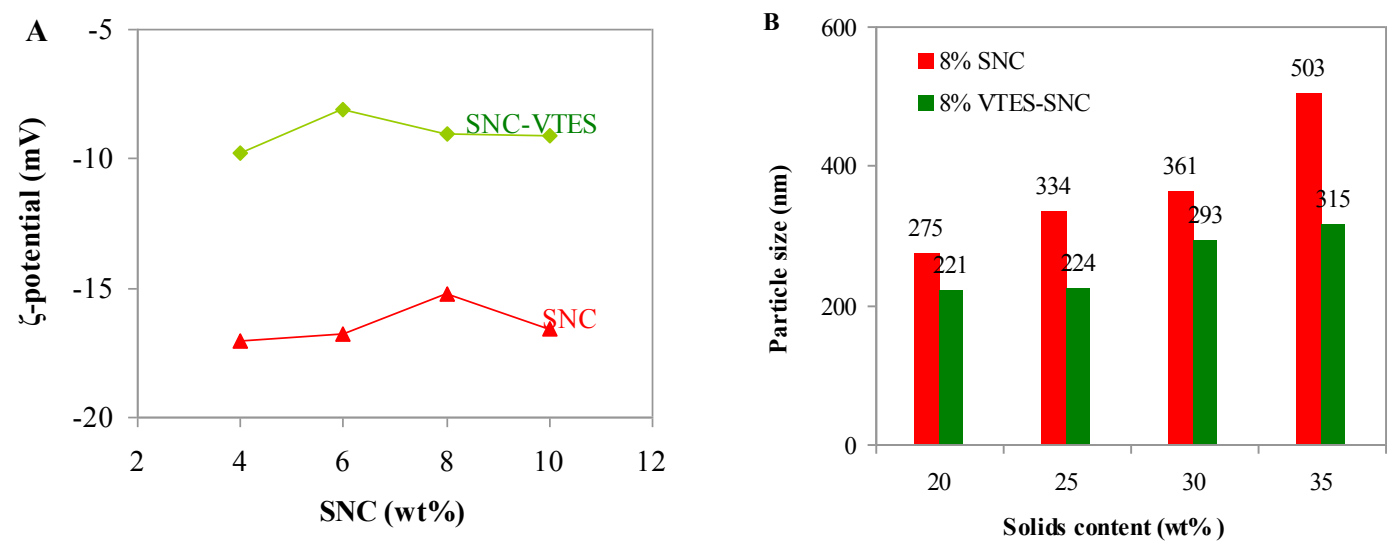

Figure 6. (A) $\zeta$-potential at $\mathrm{pH} 7$ for the PBMA latex at different SNC content, and (B) Particle size of PBMA / SNC dispersion produced by Pickering emulsion polymerization at different solid contents in presence of $8 \mathrm{wt} \%$ SNCs.

\subsection{Effect of solid content}

For practical use, latex dispersions should have a solid content at least exceeding $30 \mathrm{wt} \%$, so that their application as waterborne binders could be considered. Currently, most industrial latex dispersions are synthesized at a solid content ranging from 40 to $60 \mathrm{wt} \%$, depending on the monomer type and the morphology of the polymer particle. In most of the reported papers concerning the Pickering emulsion polymerization using solid particles, the solid content issue is overlooked and the polymerization is often performed at a low content. To address this issue, the emulsion polymerization of BMA at different solid contents was performed using both neat SNCs and VTES-SNCs (Fig. 6B). A stable dispersion with monomodal distribution was obtained in presence of VTES-SNCs as well as SNCs without any sign of particle aggregation up to a solid content of $35 \mathrm{wt} \%$. For all dispersions, smaller particle were formed when VTES-SNCs were used. At $35 \mathrm{wt} \%$ solid content, the particle size was around 500 and $300 \mathrm{~nm}$ in the presence of $8 \mathrm{wt} \%$ SNCs and VTES-SNCs, respectively. However, it is worth noting that the latex synthesized in the presence of SNCs settled down after about three weeks ofstorageat room temperature while that produced using VTES-SNCs remained stable over more than two months. At $40 \mathrm{wt} \%$, stable latex dispersions could not be produced even by operating under starved conditions, known to favor the production of high solid content latex dispersions [34].

In view of the above data, we propose the following sequence for the Pickering emulsion polymerization of BMA stabilized by SNCs: 
1) Polymer particles nucleate, similarly to conventional emulsion polymerization, by precipitation of oligomer chains formed by the reaction of radicals from initiator decomposition, with the dissolved monomer in the aqueous phase.

2) The primary particles aggregate until reaching a critical size and are stabilized by attachment of SNCs on their surface. This process is favored in the presence of VTES-SNCs due to their higher hydrophobicity (contact angle around $85^{\circ}$ against $23^{\circ}$ for neat SNCs) and the possibility of direct grafting of the oligoradical chains on VTES-SNCs by the reaction of the terminal radical with the vinyl group on the surface of VTES-SNCs.

3) The polymer particles grow by a continuous supply of butylmethacrylate from the monomer droplet by diffusion through the aqueous phase. With the increase in particle size, more SNCs are required to ensure the stabilization of the polymer particles and prevent their aggregation. The growing process continues until the depletion of the monomer droplet.

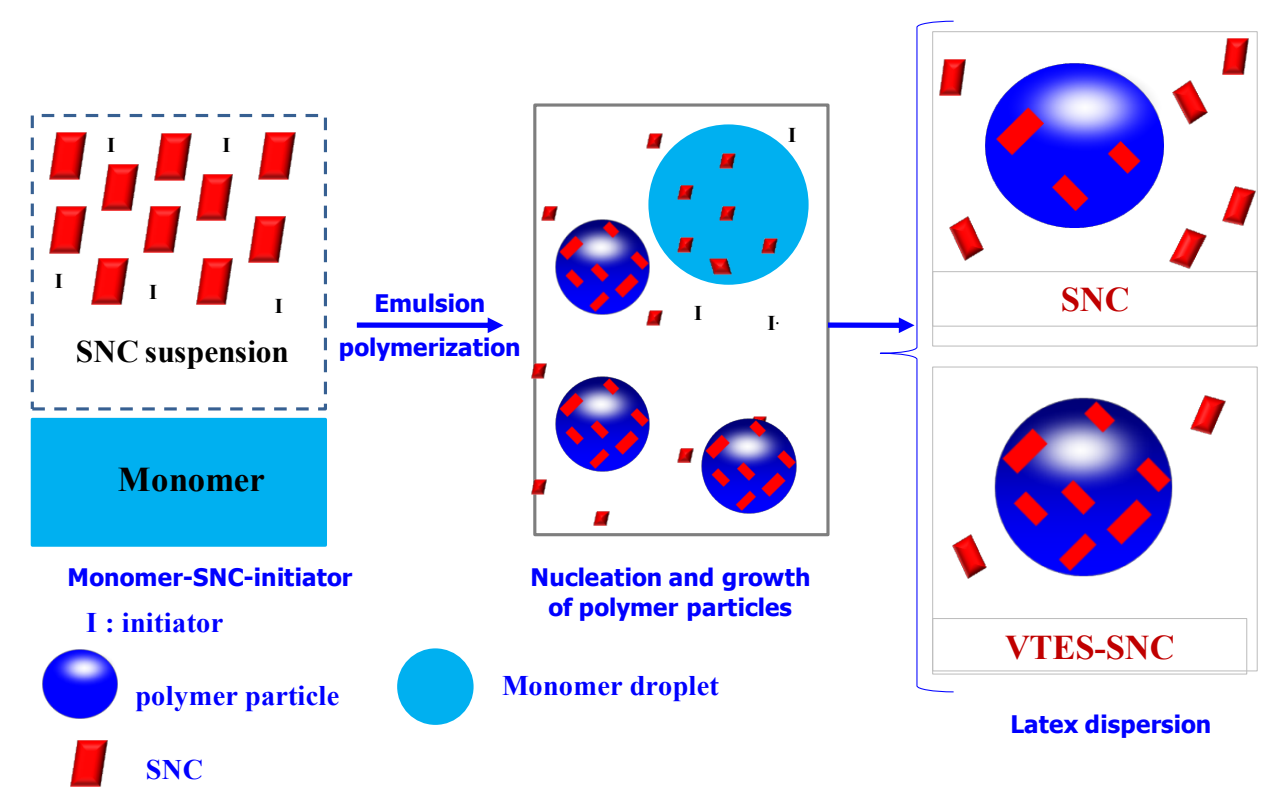

Scheme 2 Schematic illustration of the Pickering emulsion polymerization of BMA in the presence of SNCs.

Given the absence of any surfactant during the polymerization and the use of the $\mathrm{H}_{2} \mathrm{O}_{2}$ /citric acid initiator, the sole stabilization of the polymer particles will be achieved by the attachment of SNCs on the polymer particles. Accordingly, the higher the binding aptitude of SNCs on the polymer phase, the lower the particle size of the polymer dispersion. However, if we consider that a fraction of SNCs was dissolved in the form of solubilized amylopectin (Fig. 4B,C), then the following question might raise: did the dissolved fraction 
contribute to the stabilization process? To address this issue, an emulsion polymerization at $50^{\circ} \mathrm{C}$ was performed after a hydrothermal treatment of SNCs and VTES-SNCs at $70^{\circ} \mathrm{C}$ to completely dissolve SNCs into amylopectin dextrins. In both runs, a stable latex dispersion was obtained without any sign of neither aggregation nor formation of coarse lumps. However, compared to the latex dispersion prepared with neat SNCs and VTES-SNCs, a significant increase in particle size of the polymer dispersion can be noted as the SNC suspension was subjected to the hydrothermal treatment at $70^{\circ} \mathrm{C}$, as clearly shown in Fig. 7 and Table 1. This suggests that platelet-like nanocrystalline starch would have the highest efficiency as a Pickering stabilizer in emulsion polymerization. However, since a stable latex dispersion was obtained even after the complete dissolution of the of SNCs, we infer that even the amylopectin dextrins have a certain capacity to stabilize the polymer particle during emulsion polymerization, but to a lesser extent compared to the SNCs.

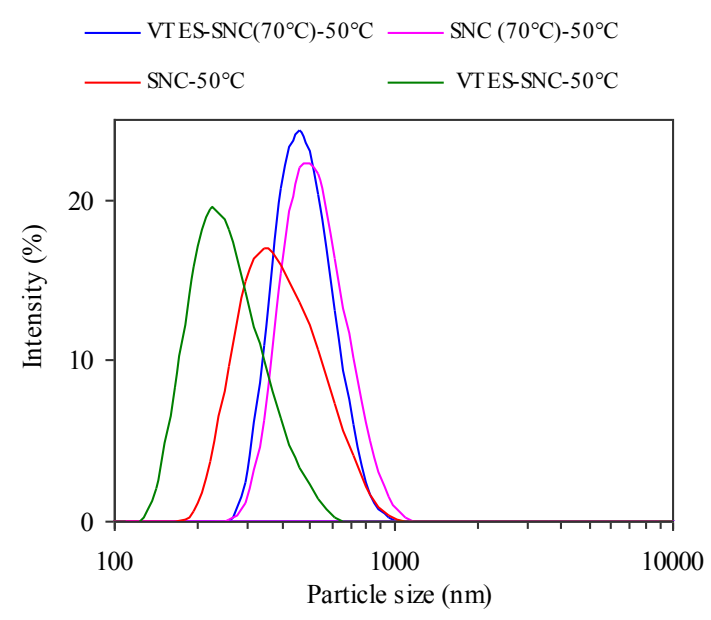

Figure 7. Particle size of the PBMA dispersion in the presence of $8 \%$ SNCs and VTES-SNCs at $50{ }^{\circ} \mathrm{C}$, at a $20 \mathrm{wt} \%$ solid content. SNC $\left(70{ }^{\circ} \mathrm{C}\right)-50{ }^{\circ} \mathrm{C}$ : SNCs submitted to a hydrothermal treatment at $70^{\circ} \mathrm{C}$ for $2 \mathrm{~h}$.

Table 1. Particle size of PBMA dispersion prepared by Pickering-emulsion polymerization in presence of $8 \%$ SNC or SNC-VTES at $20 \%$ solid content.

\begin{tabular}{cccc}
\hline Run & Stabilization & Temperature $\left({ }^{\circ} \mathrm{C}\right)$ & Particle size $(\mathrm{nm}$ \\
\hline 1 & SNC & 50 & 275 \\
2 & SNC-VTES & 50 & 221 \\
4 & SNC & 50 & 458 \\
& $\left(3 \mathrm{~h}-70{ }^{\circ} \mathrm{C}\right)$ & 50 & 385 \\
5 & SNC-VTES & 50 & \\
\hline
\end{tabular}



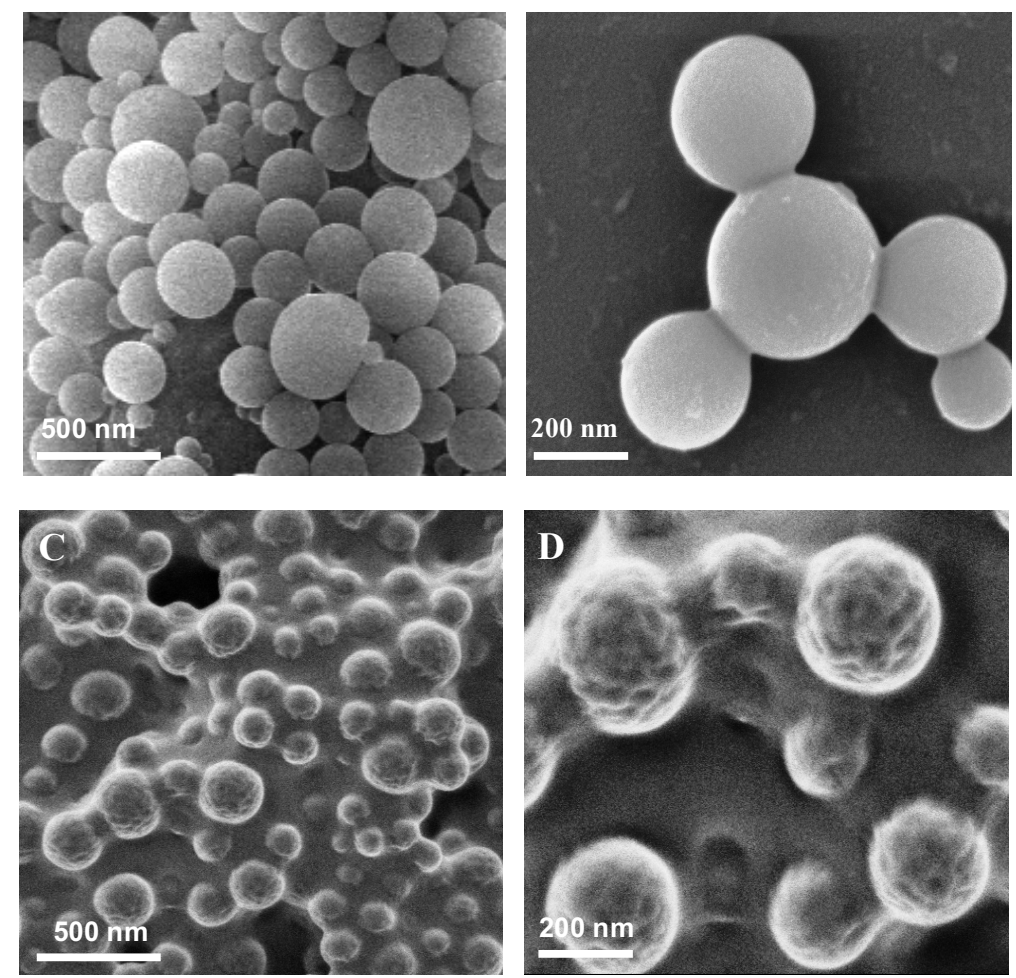

Figure 8. FE-SEM images of freeze-dried PBMA dispersion prepared in the presence of $8 \mathrm{wt} \%$ neat SNCs $(\mathrm{A}, \mathrm{B})$, and VTES-SNCs $(\mathrm{C}, \mathrm{D})$.

\subsection{Morphology of polymer particles}

The morphology of PBMA / SNC particles was observed by FE-SEM after freeze-drying of a droplet of dilute latex dispersion deposited over a silicon wafer. The images (Figs. 8A-D) showed difference in the aspect of the surface of the polymer particles. On the one hand, the polymer dispersion prepared in the presence of neat SNCs (Figs. 8A,B) contained perfectly spherical particles and that a fraction of SNCs seems to be lying outside the surface of polymer particle, meaning that only a fraction of SNCs were attached to the polymer particle after the polymerization reaction. On the other hand, for dispersion prepared in presence of VTES-SNCs (Figs. 8C,D), the polymer particles exhibited a rough surface with a raspberry-like morphology. This typical morphology was also observed in our previous work concerning the emulsion polymerization of BMA in the presence of SNCs using poly(polyethylene glycol) methacrylate (MPEG) as comonomer. However, in that work, the solid content was limited to 10 and $5 \mathrm{wt} \%$ of MPEG was added to enhance the colloidal stabilization. The size of polymer particle was around 300-400 nm and 200-300 nm for PBMA dispersion prepared in presence of neat SNCs, and VTES-SNCs, respectively, in agreement with DLS measurement. 


\subsection{Reinforcing effect of SNCs}

Given their nanometric size, platelet-morphology and crystalline structure inducing a high stiffness, the inclusion of SNCs in a polymer matrix is expected to bring about a reinforcing effect, which will result in an enhancement of the stiffness and strength of the nanocomposite film. However, this reinforcing effect strongly depends on a number of factors including; (i) the dispersion efficiency of the nanofiller, (ii) the fractal clustering in the matrix and (iii) the interfacial interaction with the polymer. To investigate how the VTES functionalization of SNCs affects the reinforcing potential of the nanocomposites, films were prepared by casting the PBMA-SNC dispersion in a Teflon mold and evaporating water at $45^{\circ} \mathrm{C}$ until complete coalescence of polymer particles and formation of homogenous translucent films. The films were analyzed by DMA by following the evolution of the storage modulus $\mathrm{E}^{\prime}$ as a function of temperature over a temperature domain spanning the glass and the rubbery domain of the polymer matrix (Fig. 9A-C).
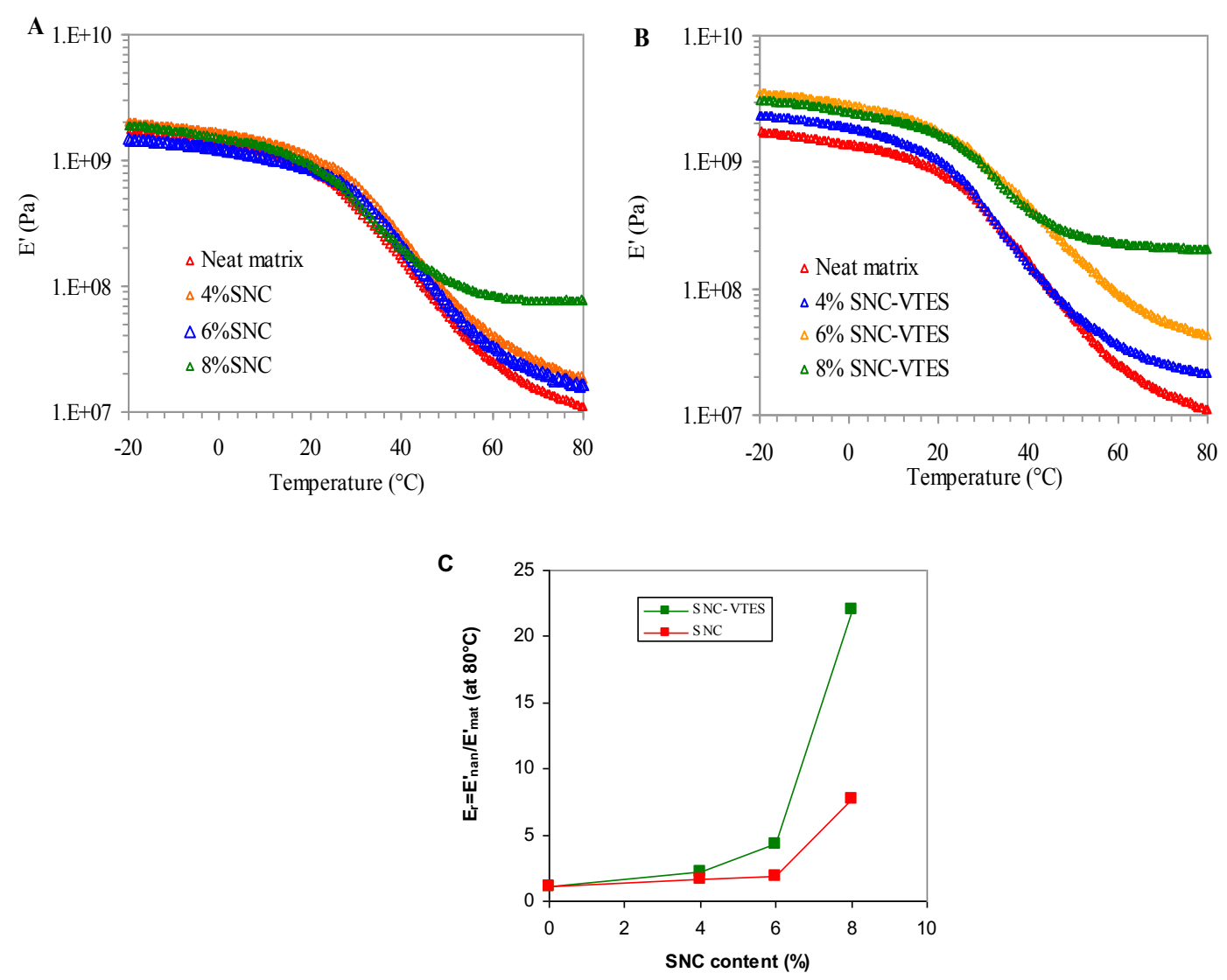

Figure 9. Storage modulus $E^{\prime}$ vs temperature for nanocomposites films produced from PBAM dispersion via Pickering emulsion polymerization in the presence of SNCs (A), VTES$\mathrm{SNCs}(\mathrm{B})$ at different contents;(C)Plot of $\mathrm{E}_{\mathrm{r}} v s \mathrm{SNC}$ content for the nanocomposite films. 
The glass transition temperature $\left(\mathrm{T}_{\mathrm{g}}\right)$ of PBMA determined from the maximum in loss factortan $\delta=E^{\prime \prime} / E^{\prime}$ with E", the loss modulus, was around $50^{\circ} \mathrm{C}$. Below the glass transition, for both SNCs and VTES-SNCs, only a small increment in modulus was noted on the nanocomposite film. At $8 \mathrm{wt} \%$ VTES-SNCs, E' reached about $2.15 \mathrm{GPa}$ at $10{ }^{\circ} \mathrm{C}$ against 1.16 $\mathrm{GPa}$ for unfilled PBMA matrix. Above $\mathrm{T}_{\mathrm{g}}, \mathrm{E}^{\prime}$ is more sensitive to the presence of SNCs, especially when VTES-SNCs were used. This could be seen from the shift of the rubbery plateau to a higher level as the SNC content is increasing. The plot of the increment in the modulus $E_{r}=\frac{E_{n a n}^{\prime}}{E_{\text {mat }}^{\prime}}$ (with $E_{\text {nan }}^{\prime}$ and $E_{\text {mat }}^{\prime}$ the storage modulus of the nanocomposite and unfilled matrix respectively measured in the rubbery region taken here at $80{ }^{\circ} \mathrm{C}$ ) versus the SNC content gives a better insight on the stiffening effect (Fig. 9C). Up to $6 \mathrm{wt} \%$ nanofiller, the presence of SNCs only resulted in a modest enhancement in $E_{r}$ with an increment in the modulus of about 3.5 in presence of $6 \mathrm{wt} \%$ VTES-SNCs. At $8 \mathrm{wt} \%$ SNCs, a marked jump in the stiffening effect was noted with an increment in $\mathrm{E}_{\mathrm{r}}$ around 20 and 9 times that of the neat matrix in presence of VTES-SNCs and SNCs, respectively. This reinforcing effect of SNCs is well known and attributed to the formation of a percolating starch nanocrystal network through hydrogen linkages among starch nanoparticle clusters [16]. The low aspect ratio of SNC compared to cellulose nanocrystals explains the higher SNCs content required to reach the setup of percolated network.

Two reasons might explain the higher reinforcing effect of VTES-SNCs. First, the better individualization of SNCs within the polymer matrix due to the more favorable attachment of VTES-SNCs to the latex particle, and second, the binding of VTES-SNCs via chemical linkage through the vinylsilane moiety, promoting a better stress transfer to the nanofiller via the continuous matrix phase.

Based on DMA analysis, there was no evidence of a meaningful evolution of the $T_{g}$ of PBMA according to whether SNC or VTES-SNCs were used.

\subsection{Pressure-sensitive adhesive application}

Pressure-sensitive adhesives (PSAs) are used for adhesive tapes and labelling for many applications, such as labels, packaging tapes, medical tapes, and automotive tapes. Among the different formulation possibilities, waterborne PSA dispersions have the merit to meet environmental regulations that ban the emission of volatile organic compounds. However, generally waterborne PSAs have poor adhesive performance due to the presence of surfactant 
that might migrate to the bonded interface [35]. One alternative to improve the adhesion is to add tackifier resins to the latex dispersion. Another alternative is the inclusion of nanofillers such as carbon nanotubes, nanoclay or nanosilica [36] and cellulose nanocrystals [37] that improve the adhesive through different mechanisms including reinforcing effects and modification of viscoelastic properties [38].
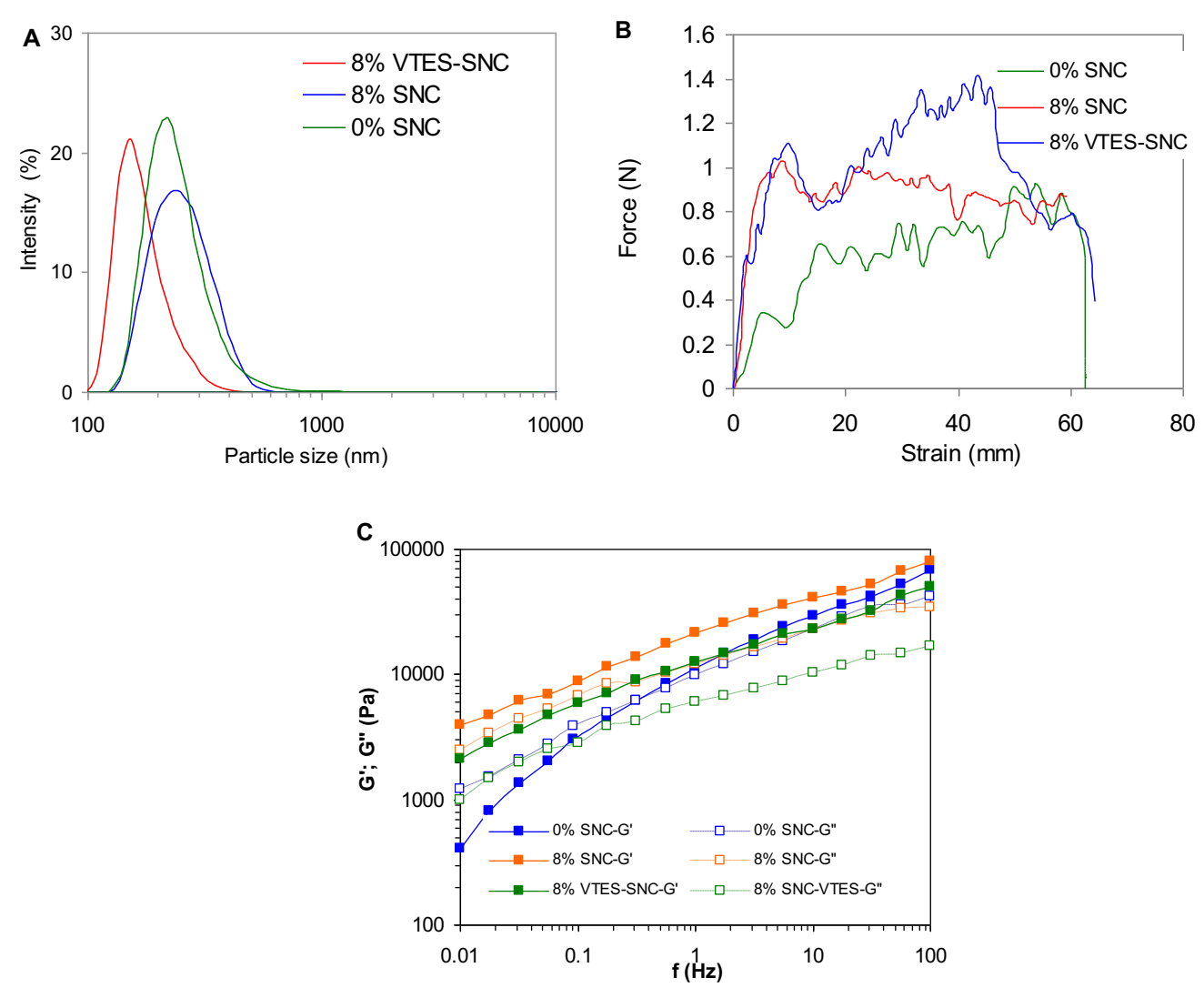

Figure 10. (A) Particle diameter distribution of the PSA dispersion; (B) peel-adhesion plot for PSA film prepared in the presence and in the absence of SNCs; (C) plot of G' and G" vs frequency of PSA films.

One might consider the use of acrylic-SNC nanocomposite dispersions as binders to produce waterborne acrylic PSAs ready for use without tackifier addition. For this purpose, an acrylic dispersion based on ethyl hexyl acrylate was synthesized by emulsion polymerization in the presence of $8 \mathrm{wt} \%$ SNCs as a sole stabilizer, using either unmodified SNCs or VTESSNCs. For comparison purposes, an acrylic PSA dispersion without SNCs was prepared following the same synthesis route, but with the addition of an anionic surfactant/MPEG to ensure colloidal stabilization during polymerization.

The particle size of the PSA dispersion produced at a $25 \mathrm{wt} \%$ solid content was around 250, 200 and $230 \mathrm{~nm}$ for PSA dispersions prepared in the presence of $8 \mathrm{wt} \% \mathrm{SNCs}, 8 \mathrm{wt} \%$ 
VTES-SNCs and without SNCs, respectively (Fig. 10A). These particle sizes are close to those obtained when the BMA monomer was used.

The peel test plot for the PSA for the different samples is given in Fig. 10B. In comparison with PSA films without SNCs, an increase in the area under the stress/strain curves was noted with the inclusion of $8 \mathrm{wt} \%$ SNCs with higher magnitude reached with VTES-SNCs, meaning that the total energy of adhesion increased when a PSA dispersion stabilized by SNCs was used, particularly in the presence of VTES-SNCs. If we consider that the first peak at low strain is due to a clean detachment of the adhesive from the probe (adhesive failure), and the elongated shoulders at higher strain are due to the formation and stretching of fibrils in the adhesive (cohesive failure), then we presume that PSA adhesive produced via SNC-stabilized acrylic dispersion has higher adhesive and cohesion strength than PSA latex produced by conventional emulsion polymerization in presence of surfactant.

The viscoelastic property of the PSA film produced by simply drying the latex dispersion at room temperature on a Teflon mold was investigated by measuring in the linear domain the evolution of storage modulus $G^{\prime}$ and loss modulus $G^{\prime \prime} v s$ frequency (Fig. 10C). On the one hand, in the PSA film from a dispersion without SNCs, a cross-over frequency (fc) was observed at which $G^{\prime}$ is equal to $G^{\prime \prime}$, meaning that the sample behave like a viscoelastic fluid at frequencies lower than $\mathrm{f}_{\mathrm{c}}\left(G^{\prime}<G^{\prime \prime}\right)$ and turned to elastic material at higher frequencies exceeding $\mathrm{f}_{\mathrm{c}}$. On the other hand, the cross-over frequency of the sample containing SNCs vanished with $G^{\prime}$ being higher than $G^{\prime \prime}$ over the whole frequency domain, which indicates the elastic properties dominated over viscous flow. However, when VTES-SNCs were used, a downward shift of both $G^{\prime}$ and $G^{\prime \prime}$ was observed in comparison with the sample prepared with SNCs, indicating a decrease in the elasticity of the nanocomposite film. It can be also seen that the magnitude of $G^{\prime \prime}$ was similar to that of the film without SNC and even lower at a frequency exceeding $0.03 \mathrm{~Hz}$.

From the rheology analysis, it became clear that the presence of SNCs, acting both as a colloidal stabilizer and nanofiller, resulted in an increase in storage moduli $G^{\prime}$ and $G^{\prime \prime}$ (for dispersion with SNCs) of the nanocomposite film, presumably due to the restriction of the flowing of polymer chain movement imparted by the presence of the nanofiller. Accordingly, the detachment of the PSA film from the substrate and the cohesive breakup of the adhesive layer would require higher energies. Moreover, the higher elastic contribution at high frequencies, $G^{\prime \prime}>G^{\prime}$, would improve the resistance to deformation at peeling conditions. These effects explain the better adhesion performances of PSA produced from Pickering emulsion polymerization of low $\mathrm{T}_{\mathrm{g}}$ monomers stabilized with SNCs. 


\section{Conclusion}

This study reports on the efficiency of SNCs to act as a stabilizer in Pickering emulsion polymerization of acrylic monomers using redox $\mathrm{H}_{2} \mathrm{O}_{2}$ /citric acid as initiator, without the addition of either surfactants or costabilizers. Stable latex dispersions with solid contents up to $35 \mathrm{wt} \%$ and SNC contents from 4 to $10 \mathrm{wt} \%$ (based on monomer) can be produced during a 4-h polymerization at $50^{\circ} \mathrm{C}$. The particle size of the polymer latex is controlled by the SNC content of with a linear dependence of the inverse diameter of the polymer particles (1/D) $v s$ SNC content, which is consistent with a stabilization being driven by a Pickering effect. The modification of SNCs with vinyltriethoxysilane was shown to increase the stabilization efficiency of SNCs allowing to reach lower particle sizes than in the presence of neat SNCs. This modification significantly enhances the reinforcing potential of SNCs, especially when the content exceeds $6 \mathrm{wt} \%$. The possibility of chemical linkage between SNCs and the polymer matrix through the vinyl silane moiety was put forward to account for this enhancement.

As a practical application of the Pickering emulsion polymerization using SNCs as stabilizer, PSA formulations based on a copolymer of ethylhexylacrylate and BMA were proposed. The presence of SNCs was shown to be beneficial of the peel strength of the PSA, mostly when VTES-SNCs were used. In addition, in the presence of VTES-SNCs, a decrease in the $G^{\prime \prime}$ loss modulus of the PSA was noted in comparison with PSA prepared without SNC or in presence of neat SNCs, implying an improvement in wetting properties of the PSA.

Since SNCs are biosourced, environment-friendly, harmless and easy-to-produce materials, their application as green Pickering stabilizers in substitution of synthetic surfactants in emulsion polymerization are promising to produce a wide range of waterborne latexes for coatings, adhesives, inks, drug delivery systems, and textiles.

\section{Acknowledgements}

This work has been partially supported by the LabEx Tec 21 (Investissements d'Avenirgrant agreement \#ANR-11-LABX-0030). LRP and CERMAV are part of Institut Carnot PolyNat (Investissements d'Avenir - grant agreement \#ANR-11-CARN-030-01). The authors acknowledge the Glyco@Alps program (Investissements d'Avenir - grant agreement \#ANR15-IDEX-02) for financial support, and thank the NanoBio-ICMG Platform (FR 2607, Grenoble) for granting access to the Electron Microscopy facility. The FE-SEM images were 
recorded at the CMTC characterization platform of Grenoble INP supported by LabEx CEMAM (Investissements d'Avenir, grant agreement \#ANR-10-LABX-44-01). We thank Rachel Martin (CMTC) for the FE-SEM observations. The PHC Utique 19G1123 is also acknowledged.

\section{References}

[1] D. LeCorre, J. Bras, A. Dufresne, Starch nanoparticles: A review. Biomacromolecules 11, (2010) 1139-1153

[2] N. Lin, J. Huang, A. Dufresne, Preparation, properties and applications of polysaccharide nanocrystals in advanced functional nanomaterials: a review, Nanoscale 4 (2012) 3274-3294

[3] C. Endes, S. Camarero Espinosa, S. Mueller, E.J. Forster, A. Petri Fink, B. Rothen Rutishauser, C. Weber, M.J.D. Clift. A critical review of the current knowledge regarding the biological impact of nanocellulose. Journal of Nanobiotechnology 14 (2016) 78-92

[4] B. Thomas, M.C. Raj, A.K. B, R.M. H, J. Joy, A. Moores, G.L. Drisko, C. Sanchez, Nanocellulose, a versatile green platform: From biosources to materials and their applications, Chemical Reviews 118 (2018) 11575-11625

[5] H.-Y. Kim, S.S. Park, S.-T. Lim, Preparation, characterization and utilization of starch nanoparticle, Colloids and Surfaces B: Biointerfaces 126 (2015) 607-620.

[6] D. LeCorre, J. Bras, A. Dufresne, Influence of native starch's properties on starch nanocrystals thermal properties, Carbohydrate Polymers 87 (2012) 658-666.

[7] O.A. Battista. In Microcrystal Polymer Science; McGraw-Hill Book Company: New York, 1975, pp 138-145.

[8] J.-P. Robin, C. Mercier, F. Duprat, R. Charbonnière, A. Guilbot. Lintnerized starches. Chromatographic and enzymatic studies of insoluble residues from acid hydrolysis of various cereal starches, particularly waxy maize starch, Starch/Stärke 27 (1975) 36-45.

[9] A. Dufresne, J.-Y. Cavaillé, W. Helbert. New nanocomposite materials: Microcrystalline starch reinforced thermoplastic, Macromolecules 29 (1996) 7624-7626.

[10] J.-L. Putaux, S. Molina-Boisseau, T. Momaur, A. Dufresne. Platelet nanocrystals resulting from disruption of waxy maize starch granules by acid hydrolysis, Biomacromolecules 4 (2003) 1198-1202.

[11] H. Angellier, L. Choisnard, S. Molina-Boisseau, P. Ozil, A. Dufresne. Optimization of the preparation of aqueous suspensions of waxy maize starch nanocrystals using a response surface methodology, Biomacromolecules 5 (2004) 1545-1551. 
[12] H. Angellier, J.-L. Putaux, S. Molina-Boisseau, D. Dupeyre, A. Dufresne. Starch nanocrystal fillers in an acrylic polymer matrix, Macromolecular Symposia 221 (2005) 95-104.

[13] H. Angellier, S. Molina-Boisseau, L. Lebrun, A. Dufresne, Processing and structural properties of waxy maize starch nanocrystals reinforced natural rubber, Macromolecules 38 (2005) 3783-3792.

[14] H. Angellier, S. Molina-Boisseau, P. Dole, A. Dufresne, Thermoplastic starch-waxy maize starch nanocrystals nanocomposites, Biomacromolecules 7 (2006) 531-539.

[15] A. Rodrigues, M. Emeje, Recent applications of starch derivatives in nanodrug delivery, Carbohydrate Polymers 87 (2012) 987-994.

[16] P. Dandekar, R. Jain, T. Stauner, B. Loretz, M. Koch, G. Wenz, C.-M. Lehr, A hydrophobic starch polymer for nanoparticle-mediated delivery of docetaxel, Macromolecular Bioscience 12 (2012) 184-194.

[17] Y. Chevalier, M.-A. Bolzinger, Emulsions stabilized with solid nanoparticles: Pickering emulsions, Colloids and Surfaces A: Physicochemical and Engineering Aspects 439 (2013) 23-34.

[18] C. Li, Y. Li, P. Sun, C. Yang, Starch nanocrystals as particle stabilisers of oil-in-water emulsions, Journal of the Science of Food and Agriculture 94 (2014) 1802-1807.

[19] B. B. Sánchez de la Concha, E. Agama-Acevedo, A. Agurirre-Cruz, L. A. Bello-Pérez, J. Alvarez-Ramírez, OSA Esterification of Amaranth and Maize Starch Nanocrystals and Their Use in "Pickering" Emulsions, Starch/Stärke 72 (2020) 1900271

[20] S.B. Haaj, W. Thielemans, A. Magnin, S. Boufi, Starch nanocrystal stabilized Pickering emulsion polymerization for nanocomposites with improved performance, ACS Applied Materials \& Interfaces 6 (2014) 8263-8273.

[21] S.B. Haaj, A. Magnin, S. Boufi, Starch nanoparticles produced via ultrasonication as a sustainable stabilizer in Pickering emulsion polymerization, RSC Advances 4 (2014) $42638-42646$.

[22] N. Nikfarjam, N. Taheri Qazvini, Y. Deng, Cross-linked starch nanoparticles stabilized Pickering emulsion polymerization of styrene in w/o/w system, Colloid and Polymer Science 292 (2014) 599-612.

[23] Y. Zhang, M.F. Cunningham, N.M.B. Smeets, M.A. Dubé, Starch nanoparticle incorporation in latex-based adhesives, European Polymer Journal 106 (2018) 128-138.

[24] S. Cummings, E. Trevino, Y. Zhang, M. Cunningham, M.A. Dubé, Incorporation of modified regenerated starch nanoparticles in emulsion polymer latexes, Starch/Stärke 71 (2019) 1800192.

[25] T. Materne, F. de Buyl, G.L. Witucki. Organosilane technology in coating applications: Review and perspectives, Dow Corning Corporation, 2012 
[26] W. Thielemans, M.N. Belgacem, A. Dufresne, Starch nanocrystals with large chain surface modifications, Langmuir 22 (2006) 4804-4810.

[27] H. Angellier, L. Choisnard, S. Molina-Boisseau, P. Ozil, A. Dufresne. Optimization of the preparation of aqueous suspensions of waxy maize starch nanocrystals using a response surface methodology, Biomacromolecules 5 (2004) 1545-1551.

[28] M. Abdelmouleh, S. Boufi, M.N. Belgacem, A.P. Duarte, A. Ben Salah, A. Gandini, Modification of cellulosic fibers with functionalized silanes: development of surface properties, International Journal of Adhesion and Adhesives 24 (2004) 43-54.

[29] H. Angellier-Coussy, J.-L. Putaux, S. Molina-Boisseau, A. Dufresne, E. Bertoft, S. Pérez. The molecular structure of waxy maize starch nanocrystals, Carbohydrate Research 344 (2009) 1558-1566.

[30] J.-L. Putaux, A. Buléon, H. Chanzy. Network formation in dilute amylose and amylopectin studied by TEM, Macromolecules 33 (2000) 6416-6422.

[31] N. Kohut-Svelko, R. Pirri, J. M. Asua, J. R. Leiza, Redox initiator systems for emulsion polymerization of acrylates. Journal of Polymer Science Part A: Polymer Chemistry 47 (2009) 2917-2927.

[32] B.P. Binks, Particles as surfactants - similarities and differences, Current Opinion in Colloid \& Interface Science 7 (2002) 21-41.

[33] H. Yotsumoto, R.H. Yoon, Application of Extended DLVO Theory: II. Stability of silica suspensions. Journal of Colloid and Interface Science 157 (1993) 434-441.

[34] S. Sajjadi, Particle formation under monomer-starved conditions in the semibatch emulsion polymerization of styrene. I. Experimental, Journal of Polymer Science Part A: Polymer Chemistry 39 (2001) 3940-3952.

[35] J. Mallégol, J.P. Gorce, O. Dupont, C. Jeynes, P.J. McDonald, J.L. Keddie, Origins and effects of a surfactant excess near the surface of waterborne acrylic pressure-sensitive adhesives, Langmuir 18 (2002) 4478-4487.

[36] M. Khalina, M. Sanei, H.S. Mobarakeh, A.R. Mahdavian, Preparation of acrylic/silica nanocomposites latexes with potential application in pressure sensitive adhesive, International Journal of Adhesion and Adhesives 58 (2015) 21-27.

[37] Z. Dastjerdi, E.D. Cranston, M.A. Dubé, Pressure sensitive adhesive property modification using cellulose nanocrystals, International Journal of Adhesion and Adhesives 81 (2018) 36-42.

[38] J.-K. Oh, C.-H. Park, S.-W. Lee, J.-W. Park, H.-J. Kim, Adhesion performance of PSAclay nano-composites by the in-situ polymerization and mechanical blending, International Journal of Adhesion and Adhesives 47 (2013) 13-20. 